\title{
Modeling of the Midwater Trawl Dynamical Behavior Based on $\mathbf{R}$ Language
}

\author{
Li Yuwei ${ }^{1,2,3,4}$, Zou Xiaorong ${ }^{1,2,3,4,{ }^{*}, \text { Zhang Xinfeng }}{ }^{1,2,3,4}$, Zhang Min ${ }^{1,2,3,4}$, Chen Xinjun ${ }^{1,2,3,4}$, Song Liming ${ }^{1,2,3,4}$, Zhou \\ Yingqi $i^{1,2,3,4}$ \\ ${ }^{1}$ College of Marine Sciences, Shanghai Ocean University, Shanghai 201306, China \\ ${ }^{2}$ The Key Laboratory of Sustainable Exploitation of Oceanic Fisheries Resources, Ministry of Education, Shanghai \\ Ocean University, Shanghai 201306, China \\ ${ }^{3}$ National Engineering Research Center for Oceanic Fisheries, Shanghai Ocean University, Shanghai 201306, China \\ ${ }^{4}$ Collaborative Innovation Center for Distant-water Fisheries, Shanghai, 201306, China \\ e-mail: ywli@shou.edu.cn, xrzou@shou.edu.cn
}

\begin{abstract}
The midwater trawl is used widely in many oceanic fisheries. In this study, the midwater trawl dynamical behavior was simulated and modeled with the mathematical mechanic equations based on $R$ language platform. A lump-mass and spring method was used to build the physical model. The huge mathematical stiff differential equations were solved by an implicit algorithm. On the $R$ platform, the programming, simulation and visualization of the model were done quickly. The three-dimensional dynamical behavior of the midwater trawl in steady flow was presented successfully and the effectiveness of this method was verified. And the results were accord with the actual measured data in the ocean.
\end{abstract}

Keywords-component; midwater trawl;meshes grouping; lumped mass method;implicit algorithm;numerical simulation

\section{INTRODUCTION}

Up to now, the midwater trawl net is one of the most important fishing gears in the world capture fisheries. The target species of the midwater trawls are quite varied. As for the midwater trawls, they should be suitable for the behavior habits of fishing targets, also required to reduce their drag for cut down fishing boat fuel consumptions in order to improve the fishing efficiency and economic benefits, so the research on the performance of the midwater trawl net is important.

In the past two decade, the performance research of the midwater trawl has based on the water tank experiments, field measurement and numerical simulation. But, the nonlinear equations coming from the mechanical equations of different fishing gears, are so different to solve. Few studies have conducting the trawl net by numerical simulation methods. Sun et al has used the twodimensional lumped mass models to describe the dynamical behavior of the midwater trawl system [1]. Takagi et al. applied the lumped mass method in simulating the fishing gear system by subdividing the elements of fishing gears [2]. Priour et al. used the finite element method with triangular elements to simulate the trawl net shapes [3-4]. Zhu et al. applied the lumped mass method to model the dynamical behavior of supple nets and solve the nonlinear equations with Runge-kutta method [5].Lee et al. used the lumped mass method to describe the midwater trawl gear by an implicit calculation method to solve the nonlinear differential equations [6].

But, the computation of these models are too complex and very different to understand. In these models, the coordinated systems used in the computation are most based on the local coordinate systems need to be converted. The explicit method are usually used to solve these nonlinear differential equations. So, the solution of equations are instability and inaccuracy and the time step is greatly limited by the stiffness of the flexible systems [7].

$\mathrm{R}$ is a scripting language for statistical data manipulation and space analysis. It was inspired by, and developed from the statistical language $S$ developed by AT\&T [8]. It is free and many people are contributing to its development. Due to the high calculation performance, Berend Hasselman has developed a Package 'nleqslv' to solve these nonlinear and stiff equations of the flexible systems [9]. And it can be used to some simple initial value problems of the ordinary differential equations. But for the higher-order and stiff differential equations, it shows some weakness. Zhang et al described the part behavior of purse seine using $\mathrm{R}$ language [10].

But, most fishing gear studies focused or involved in statics analysis and fishing gear model tests etc., lack of dynamic performance of fishing gear study and the application of computer simulation technology, including CAD for simulation for fishing gears. In this paper, we established the mathematics-mechanical models with the lump-mass and spring method to simulate the dynamical behavior of the midwater trawl. The $\mathrm{R}$ language was used to compile the complicated mathematical computation with the implicit method. The visualization of the $3 \mathrm{D}$ in the equilibrium state was presented by the R package ' $3 \mathrm{D}$ Real-Time Visualization Device System for R (RGL)'. The results of calculation were correspond with the actual measured data in the ocean. The validity of this method was verified.

\section{MATERIALS AND METHODS}

\section{A. Numerical Modeling}

The midwater trawl systems is composed of cables, nettings and accessory [Fig .1]. The cables include warp, hand rope, head line, footrope, sideline, cross ropes and belly lines. The accessory include otter board, heavy 
hammer and soft canvas. For the midwater trawl, the heavy hammer and soft canvas are used to make the net open vertically, the opening force from otter board is used to make the net open horizontally, changing the warp length or trawling speed is used to make the control and adjustment of the net position. By these above measures, the aimed fishing can be done. For this complex fishing gears structure, in order to do dynamical numerical simulation, we must consider the structural features, so the trawl systems can be divided into rigid body and flexible body. Then, otter board, heavy hammer and floats can be considered as rigid bodies; cables, soft canvas and nettings can be considered as flexible bodies.

The lump-mass and spring method was used to build the model. In our model, the rigid bodies were considered as mass points. The flexible bodies were composed of many lumped mass points interconnected by springs without mass. The lumped mass points were set at each knot, the midpoint of each mesh bar and each rope element. In order to simplify the model, an assumption was that no external force exist on the spring. There only exists the forces of tension force acted on the spring, and gravity, buoyancy and hydrodynamic force (drag force and lift force) acted on the mass points[11-13].

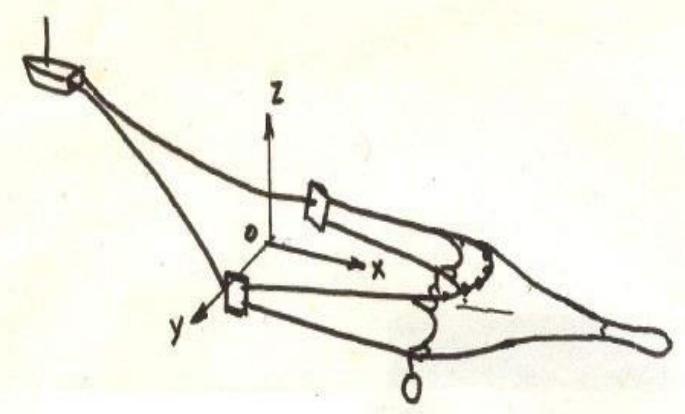

Figure 1. The schematic diagram of midwater trawl system

\section{B. Mathematics-mechanical Model}

Based on the Newton second law, the motion of point $\mathrm{i}$ could be described as follows:

$$
M_{i} a=\stackrel{1}{T}+\stackrel{1}{F}+\stackrel{1}{W}+\stackrel{1}{B}
$$

Where $a$ is the acceleration vector of point $\mathrm{i} ; T$ is the tension force acting on point i; $F$ is the hydrodynamic force including drag force and lift force; $W$ is the gravity force; $B$ is the buoyancy force, and $M$ is the mass of point $i$ including the added mass of $i$.

There is a spring without mass between neighboring points. The tension $\mathrm{T}$ based on the Hooke' law, can be described as

$$
\left|T_{i j}\right|= \begin{cases}k\left(l_{i j}-l_{0 i j}\right) & l_{i j}>l_{0 i j} \\ 0 & l_{i j} \leq l_{0 i j}\end{cases}
$$

Where, $k$ is the stiffness of the spring. $l_{0 i j}$ and $l_{i j}$ are the original length and deformed length between mass points $\mathrm{i}$ and $\mathrm{j}$, respectively.
For the mesh knot can be considered as a spherical shape [Fig .2], the hydrodynamic force can be described as the following equations:

$$
\mathbf{F}=\frac{1}{2} C_{D} \rho S_{1} \mathbf{V}^{2}
$$

Where, $\mathbf{F}$ is the hydrodynamic force, $C_{D}$ is the hydrodynamic coefficient, $\rho$ is the water density, $S_{1}$ is the projected area of mesh knot, $\mathbf{V}$ is the resultant speed vector.

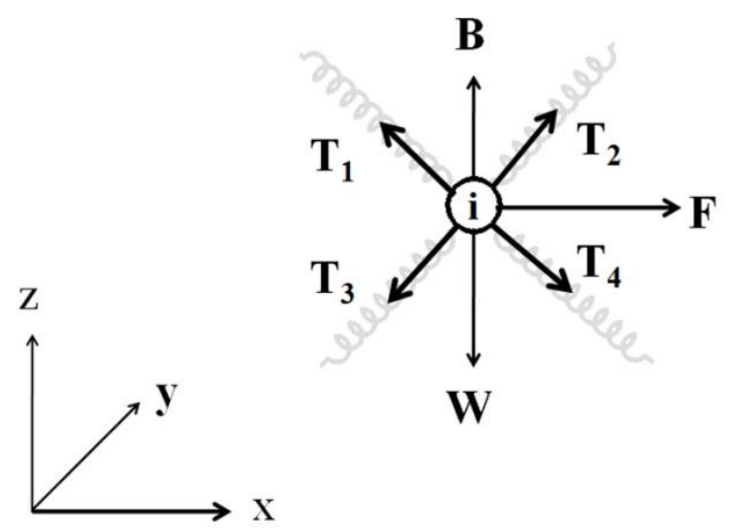

Figure 2. The schematic diagram of the mesh knot

For the mesh bar and the rope elements can be considered as cylindrical elements, it was assumed that the angle of the bar between the resultant speed vector was $\alpha$ [Fig .3].

The hydrodynamic force could be divided into lift force and drag force. The lift force and drag force of mass point i can be expressed as

$$
\begin{aligned}
& \mathbf{F}_{L}=\frac{1}{2} C_{L} \rho S_{2} \mathbf{V}^{2} \\
& \mathbf{F}_{D}=\frac{1}{2} C_{D} \rho S_{2} \mathbf{V}^{2}
\end{aligned}
$$

Where, $C_{L}$ and $C_{D}$ are the lift force coefficient and drag force coefficient determined by the angle $\alpha$ [Fig .3], separately. $\rho$ is the water density, $S_{2}$ is the projected area of structure, $\mathbf{V}$ is the resultant speed vector.

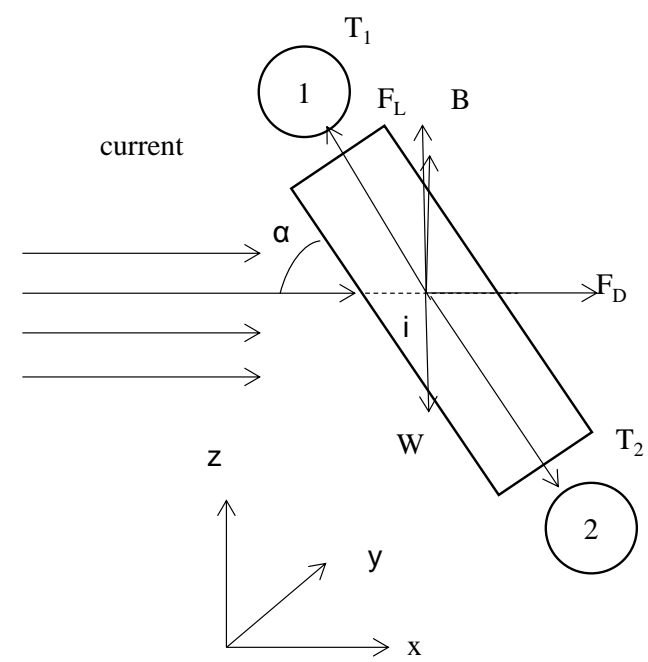

Figure 3. The schematic diagram of numerical model of cable element 
The gravity and buoyancy of mass point $\mathrm{i}$ can be described as

$$
\begin{array}{r}
\mathbf{W}=\rho_{i} V_{i} \mathbf{g} \\
\mathbf{B}=\rho_{i} V_{i} \mathbf{g}
\end{array}
$$

Where, $\rho_{i}$ is the material density, $V_{i}$ is the volume of mass point $\mathrm{i}, \mathbf{g}$ is the acceleration of gravity.

The added mass of point $i$ can be shown in the following formula

$$
m_{a}=C_{m} \rho V_{i}
$$

Where, $C_{m}$ is the added mass coefficient.

For the point $\mathrm{i}$, the motion equations can be expressed

$$
\left\{\begin{array}{l}
M_{i}=\left(\mathbf{T}_{\mathbf{x}}+\mathbf{F}_{D \mathbf{x}}+\mathbf{F}_{L \mathbf{x}}\right) \\
M_{i}=\left(\mathbf{T}_{\mathbf{y}}+\mathbf{F}_{D \mathbf{y}}+\mathbf{F}_{L \mathbf{y}}\right) \\
M_{i}=\left(\mathbf{T}_{\mathbf{z}}+\mathbf{F}_{D \mathbf{z}}+\mathbf{F}_{L \mathbf{z}}+\mathbf{B}_{\mathbf{i}}-\mathbf{W}_{\mathbf{i}}\right)
\end{array}\right.
$$

Equation (9) can be changed into ordinary differential equations

$$
\begin{aligned}
& =f_{2}\left(x_{i}, y_{i}, z_{i}, \underset{i}{\&}, \underset{1}{\&}, x_{1}, y_{1}, z_{1}, x_{2}, y_{2}, z_{2} ; t\right) \\
& =g_{2}\left(x_{i}, y_{i}, z_{i}, \underset{1}{\&}, x_{1}, y_{1}, z_{1}, x_{2}, y_{2}, z_{2} ; t\right) \\
& =h_{2}\left(x_{i}, y_{i}, z_{i}, \underset{i}{\&}, \underset{1}{\&}, x_{1}, y_{1}, z_{1}, x_{2}, y_{2}, z_{2} ; t\right)
\end{aligned}
$$

\section{Simulation}

For modelling, we used virtual matrix to establish the data structure and geometric modeling [14].The equations solution could be solved as an initial value problem. The methods can be divided into the explicit method and the implicit method. The equation of this flexible system is stiff equations, which are sensitive to time step. Due to the time step is sensitive and must be very small, the explicit method cannot be suitable for solving theses stiff equation. The stiff equations solving by the implicit method are more stable and accurate than the explicit method. The non-self-starting Heun method(seen 11) is one kind of representative implicit methods [15]. In this paper, the non-self-starting Heun method is used to solve the differential equation. This method could guarantee the stability and accuracy.

$$
y_{i+1}^{j}=y_{i}^{m}+\frac{f\left(x_{i}, y_{i}^{m}\right)+f\left(x_{i+1}, y_{i+1}^{j-1}\right)}{2} h
$$

Equation (10) is implicit and high-order ordinary differential equations. In order to resolve directly in $\mathrm{R}$, we define $\underset{i}{\&}=q_{i}, \underset{i}{\&}=u_{i}, \underset{i}{\&}=h_{i}$. Then could be converted to first-order differential equations

$$
\begin{aligned}
& \underset{i}{\&}=f_{1}\left(x_{i}, y_{i}, z_{i}, q_{i}, x_{j}, y_{j}, z_{j} ; t\right) \\
& \iota_{i}^{\&}=g_{1}\left(x_{i}, y_{i}, z_{i}, u_{i}, x_{j}, y_{j}, z_{j} ; t\right) \\
& \boldsymbol{H}_{i}^{\mathrm{k}}=h_{1}\left(x_{i}, y_{i}, z_{i}, h_{i}, x_{j}, y_{j}, z_{j} ; t\right) \\
& \underset{i}{\&}=f_{2}\left(x_{i}, y_{i}, z_{i}, q_{i}, u_{i}, h_{i}, x_{1}, y_{1}, z_{1}, x_{2}, y_{2}, z_{2} ; t\right) \\
& \iota_{i} \&=g_{2}\left(x_{i}, y_{i}, z_{i}, q_{i}, u_{i}, h_{i}, x_{1}, y_{1}, z_{1}, x_{2}, y_{2}, z_{2} ; t\right) \\
& H_{i}^{\&}=h_{2}\left(x_{i}, y_{i}, z_{i}, q_{i}, u_{i}, h_{i}, x_{1}, y_{1}, z_{1}, x_{2}, y_{2}, z_{2} ; t\right)
\end{aligned}
$$

The backward Euler method was used to calculate (12) and (13). There is an assumption that the total time $t$ of the motion of rope is divided into $\mathrm{T}$ steps, $\mathrm{n} \leqslant \mathrm{T}$. And $\mathrm{dt}$ is time step and $\mathrm{dt}=\mathrm{t}[\mathrm{n}+1]-\mathrm{t}[\mathrm{n}]$. Hence the improved euler method was used to solve the (12) and (13). And the improved euler method could be expressed as

$$
\begin{aligned}
& x_{i}[k+1]^{[j+1]}=x_{i}[k]+\frac{d t}{2} \times\left(q_{i}[k]+q_{i}[k+1]^{[j]}\right) \\
& y_{i}[k+1]^{[j+1]}=y_{i}[k]+\frac{d t}{2} \times\left(u_{i}[k]+u_{i}[k+1]^{[j]}\right) \\
& z_{i}[k+1]^{[j+1]}=z_{i}[k]+\frac{d t}{2} \times\left(h_{i}[k]+h_{i}[k+1]^{[j]}\right) \\
& q_{i}[k+1]^{[j+1]}=q_{i}[k]+\frac{d t}{2} \times\left(f_{i}(k)+f_{i}(k+1)^{[j]}\right) \\
& u_{i}[k+1]^{[j+1]}=u_{i}[k]+\frac{d t}{2} \times\left(g_{i}(k)+g_{i}(k+1)^{[j]}\right) \\
& h_{i}[k+1]^{[j+1]}=h_{i}[k]+\frac{d t}{2} \times\left(h_{i}(k)+h_{i}(k+1)^{[j]}\right)
\end{aligned}
$$

Where $m$ is the $j$-th step of iterations for implicit euler iterative computation.

We used this algorithm to compile the program and calculate the models in the $\mathrm{R}$ platform. The Think Workstation D10 was used to solve all equations at all given points. Then the 3D dynamical behavior and tension loads can be attained.

\section{EXPERIMENTAL RESULTS}

After modeling and programming the midwater trawl net in steady flow using mechanical equations and implicit algorithm in $\mathrm{R}$ platform. The graphical package of $\mathrm{R}$ i.e. 3D Real-Time Visualization Device System for R (RGL) was used to visualize the results. The package is so powerful that it can display the three-dimensional dynamical behavior of the midwater trawl net vividly. The results of the simulation and visualization presented lively in the equilibrium state (Fig .4, 5 and 6) and the equilibrium state were accord with the actual measured data in the ocean. Due to space limitation, we can only exhibit the three-dimensional space shape of the midwater trawl net under the sea water. Zhang et al. researched the part behavior of purse seine using half-implicit algorithm [10].

In the equilibrium state, the net opening was about $105 \mathrm{~m}$, the net horizontal spreading was about $113 \mathrm{~m}$, the otter board horizontal spreading was about $151.89 \mathrm{~m}$. These parameters were very consist with the actual measured data.

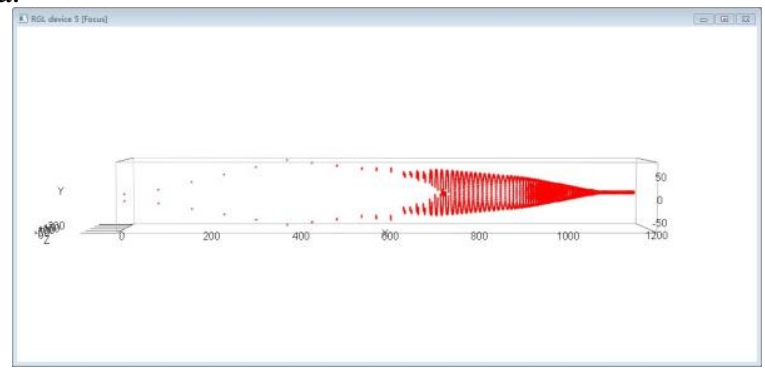

Figure 4. The XY plane figure of the midwater trawl 


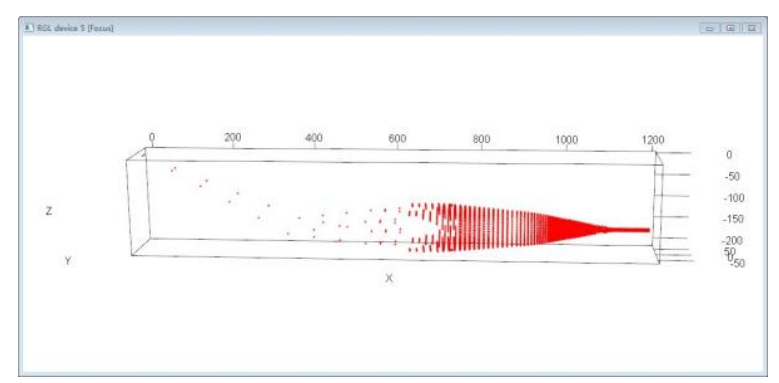

Figure 5. The $\mathrm{XZ}$ plane figure of the midwater trawl

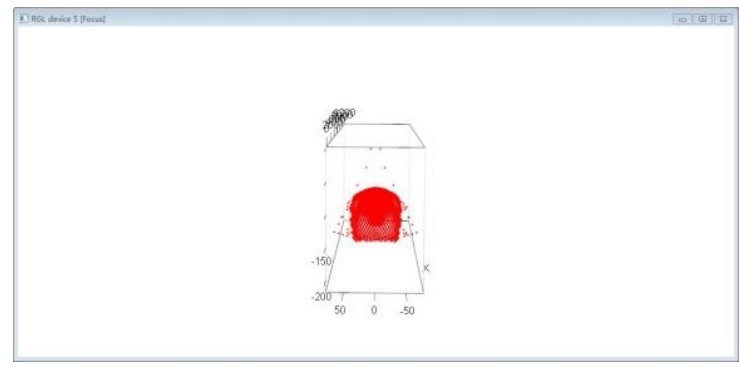

Figure 6. The YZ plane figure of the midwater trawl

\section{DISCUSSIONS}

The midwater trawl system is a soft flexible system. Its shape and tension distribution are changing and continually interacted. When some forces load on the ropes, the forces and tension in the system could change due to the change of their shape, so more time steps are need during simulation calculation period. When running the mathematical model, the stabilization is the most difficult. However, the model predicated successfully the 3D shape and tension distribution of the system in a reasonable time length. The results show that where the load highly concentrated in the system which can be an important reference during fishing gear design and operation.

\section{CONCLUSION}

In order to model the midwater trawl in steady flow, the lump-mass and spring method based on this paper was applied. The numerical model based on this method can predict the three-dimensional shape of the midwater trawl and the crucial parameters can be estimated accurately. The results show that this method can simulate other fishery equipment and engineering. Through this study, we can get more information about some more complex engineering systems such as net cages and purse seine systems consist of millions meshes and estimate the shape of space and tension loads.

\section{ACKNOWLEDGMENT}

This work was supported by: 1) The Youth Scholars of Shanghai Higher Education Institutions (A1203514001014); 2) Special funding for the development of science and technology of Shanghai Ocean University (A2020915200002); 3) The Open Foundation from Key Laboratory of Marine Fishery Equipment and Technology of Zhejiang (MFET201409); 4) PhD research startup foundation of Shanghai Ocean University (A0209130105418); 5) Key technology in high efficient utilization of Chilean jack mackerel (Trachurus murphyi) resources (No. 2012AA092301); 6) Key technology in pelagic tuna's purse seine fishing and ultralow temperature preservation (No. 2012AA092302);7) Technical development in fishing and processing of squid resources (No. 2012AA092303).

\section{REFERENCES}

[1] X. F. Sun, Y. Yin,Y. C. Jin, X. Y. Zhang, and X.F.Zhang, "The modelingofsingle-boat,mid-water trawl systems for fishing simulation," Fisheries Research, Vol.109, Apr 2011, pp. 7-15, doi:10.1016/j.fishres.2010.12.027.

[2] T. Takagi, T. Shimizu, K. Suzuki, T. Hiraishi, and K. Yamamoto, "Validity and layout of "NaLA": a net configuration and loading analysis system," Fisheries Reseach, Vol. 66, No. 2-3, Feb 2004 , pp. 235-243, doi:10.1016/S0165-7836(03)00204-2

[3] D. Priour, "Analysis of nets with hexagonal mesh using triangular element,"Numerical methods in engineering, Vol. 56, No. 12, Mar 2003, pp. 1721-1733, doi: 10.1002/nme.635.

[4] D. Priour, A Finite Element Method for Netting. Application to Fish Cagesand Fishing Gear. Dordrecht: Springer Netherlands, 2013.

[5] L.X Zhu, Z.L. Liang, L.Y. Huang, and F.F. Zhao, "Numerical simulation of dynamic response of supple nets, "China ocean Engineering, Vol. 20, No. 3,Sep 2006, pp.443-456.

[6] J. H. Lee, L. Karlsen, and C. W. Lee, "A method for improving the dynamic simulation efficiency of underwater flexible structures by implementing non-active points in modelling," ICES JOURNAL OF MARINE SCIENCE, Vol. 65, No. 9, 2008, pp. 1552-1558, doi: 10.1093/icesjms/fsn 126

[7] J. Lee, C.W. Lee, M.Y. Choe, and G.Ho. Lee, "Applying Fishinggear Simulation Software to Better Estimate Fished Space as Fishing Effort," Fisheries and aquatic sciences ,Vol. 14, No. 2, May 2011, pp. 138-147,doi:10.5657/FAS.2011.0138.

[8] N. Matloff, THE ART OF RPROGRAMMING. San Francisco:No Starch Press, 2011

[9] http://cran.csie.ntu.edu.tw/web/packages/nleqslv/index.html.

[10] X. F. Zhang, M. Wang, and L. X. Xu, "Modeling and visualization of part behavior of purse seine using R language," Proceedings 4th International Congress on Image and Signal Processing (CISP 2011), IEEE Computer Society, Oct.2011, pp.2013-2016.doi: 10.1109/CISP.2011.6100612.

[11] H.T.Thai, and S. E.Kim, "Nonlinear static and dynamic analysis of cable structures," Finite Elements in Analysis and Design, Vol. 47, No. 3, Mar 2011, pp. 237-246, doi:10.1016/j.finel.2010.10.005.

[12] S.K,and M. Mojdis, "Time-dependent analysis of cable domes using a modified dynamic relaxation method and creep theory," Computers and Structures, Vol. 125, Sep 2013, pp. 11-22, doi: 10.1016/j.compstruc.2013.04.019.

[13] Z.W.Wang,and T.J. Li,"Nonlinear dynamic analysis of parametrically excited space cable-beam structures due to thermal loads," Engineering Structures, Vol. 83, No. 15, Jan 2015, pp. 5061, doi: 10.1016/j.engstruct.2014.11.001.

[14] [X. F. Zhang, D. Cao, L. M. Song, X. R. Zou, X. J. Chen, L. X. Xu, et al, "Application of whole-implicit algorithm and virtual neural lattice in pelagic longline modeling", Proceedings of the 8th International Conference on Natural Computation(ICNC 08), IEEE Press, May. 2012, pp. 2616-2619,doi: 10.1109/FSKD.2012.6234063.

[15] C. Canale and R. Canale, Numerical Methods for Engineers 6th Edition.New York: McGraw-Hill Science, 2009 